ISSN 1014-4874

DOI : http://dx.doi.org/10.4314/rj.v27i1.2

\title{
Static Equilibrium Configurations of Charged Metallic Bodies
}

Joseph Nzabanita, National University of Rwanda, PO. Box : 117 Butare-Rwanda, jnzabanita@nur.ac.rw Magnus Herberthson, Linköping University, 58183 Linköping-Sweden, maher@mai.liu.se Bengt Ove Turesson, Linköping University, 58183 Linköping-Sweden, betur@mai.liu.se Froduald Minani, National University of Rwanda, PO. Box : 117 Butare-Rwanda, fminani@nur.ac.rw

\begin{abstract}
When charged particles are placed on an uncharged metallic body, the charged particles redistribute themselves along the surface of the body until they reach a point or a configuration that no net tangential force is experienced on each particle. That point is referred to as electrostatic equilibrium configuration or simply as static equilibrium configuration. One of the properties which a metallic body possesses at static equilibrium configuration is among others that the distribution of charges is such that the potential energy is minimized. In this paper we developed a simple numerical scheme to determine the static equilibrium configuration of charged metallic bodies by minimizing the potential energy function. The method developed has some advantages; it combines the general theory and the physical meanings nested in the mathematical model and this has a positive implication on the computational aspect. For numerical simulations we considered the case of ellipsoids. Numerical solutions were produced, presented and discussed.
\end{abstract}

Key words: Static equilibrium, charged metallic body, potential energy, projected gradient method.

\section{Introduction}

The society of today relies on electromagnetic devices and systems: television, radio, internet, microwave ovens, mobile telephones, satellite communication systems, radar systems, electrical motors, electrical generators, computers, microwave filters, lasers, industrial heating devices, medical imaging systems, electrical power networks, transformers and many more (Bondeson et al., 2005). Each of these examples is used in a broad range of situations. As an example, take radar. It is employed for fire control, weather detection, airport traffic control, missile tracking, missile guidance, speed control, traffic safety, etc. Undoubtedly, electromagnetic phenomena have a very profound impact on contemporary society (Bondeson et al., 2005). Therefore more studies for the understanding of 
electromagnetic phenomena is highly needed. This paper studied one of these phenomena, the static equilibrium configurations of charged metallic bodies.

The mathematical model of the problem that we investigate in this paper can be formulated in the following terms. Consider a metallic body with surface $S$. On the surface $S$ of the body, one has put $N$ charged particles. Denote the particles by $p_{j}, 1 \leq j \leq N$, and assume that their charges and positions are $q_{j}>0$ and $\vec{r}_{j} \in S, \quad 1 \leq j \leq N$, respectively. Since all charges are positive, all electrostatic forces are repulsive, and hence the charges will try to become as well separated as possible. The charges consider themselves well separated if their energy is low as possible (Wayne, 2002). For this particular problem, we want to choose the positions of the charges so that the potential energy

$$
U\left(\vec{r}_{1}, \ldots, \vec{r}_{N}\right)=\frac{1}{2} \sum_{k=1}^{N} \sum_{j=1, j \neq k}^{N} \frac{q_{j} q_{k}}{\left|\vec{r}_{j}-\vec{r}_{k}\right|}
$$

is minimized. In the formula (1), |.| stands for the Euclidean norm and all quantities are expressed in CGS electrostatic units. In CGS system, the units of length, mass and time are the centimeter, the gram, and the second, respectively. The formula (1) is a general expression for the potential energy of an arbitrary system of many point charges. It plays a central role in this paper, and hereafter, we give a brief description of how it can be obtained. More details can be found in (Baldassare, 1991) or in (Ohanian, 2006).

Suppose that we have a collection of $N$ point charges, with charges $q_{j}$, $1 \leq j \leq N$, at positions $\vec{r}_{j}$. What is the potential energy of this charge distribution? That is, how much work must you do to bring all these charges to their positions, starting with all of them infinitely separated? To calculate this amount of work, let us imagine that you bring the charges to their positions in succession: first, you bring $q_{1}$ to $\vec{r}_{1}$ (from infinity), then you bring $q_{2}$ to $\vec{r}_{2}$, and so on. When you bring the charge $q_{\mathrm{j}}$ to join the $j-1$ preceding charges, the potential produced by the latter is

$$
V(\vec{r})=\sum_{k=1}^{j-1} \frac{q_{k}}{\left|\vec{r}-\vec{r}_{k}\right|} .
$$

The increase in potential energy that occurs when you bring in charge $q_{\mathrm{j}}$ to $\vec{r}_{j}$ is therefore

$q_{j} V\left(\vec{r}_{j}\right)=\sum_{k=1}^{j-1} \frac{q_{j} q_{k}}{\left|\vec{r}_{j}-\vec{r}_{k}\right|}$. 
The total increase in potential energy that occurs when you bring all the charges to their positions is then

$$
U\left(\vec{r}_{1}, \ldots, \vec{r}_{N}\right)=\sum_{j=1}^{N} q_{j} V\left(\vec{r}_{j}\right)=\sum_{j=1}^{N} \sum_{k=1}^{j-1} \frac{q_{j} q_{k}}{\left|\vec{r}_{j}-\vec{r}_{k}\right|} .
$$

This expression is simply the sum of all the mutual potential energies for all possible pairs of charges $q_{\mathrm{i}}$ and $q_{\mathrm{j}}$. Observing that each term of the summation in equation (4) occurs twice in the summation in equation (1), equation (1) and (4) are equivalent.

\section{Method}

It is clear that the problem at hand belongs to a broad class of optimization problems. More specifically it is a non linear constrained optimization problem. For more about optimization problems see (Nash \& Sofer, 2009) or (Wenyu \& Ya-Xiang, 2006). In general, a big issue to handle non linear problems is the lack of methods that can be used to get analytical solutions and thus most of the time numerical methods are used instead. Most of algorithms for optimization problems seek a local minimizer (Iusem, 2003), that is, a point that is only locally minimal, which means that it minimizes the objective function among feasible points that are near it. The projected gradient method is also of that kind. However it has been proved in (Iusem, 2003) that for the case in which the objective function is convex, which is the case for our problem, under the only assumption of existence of solutions they are global minimizers. In this paper we develop a numerical scheme, a variant of the projected gradient method, that intends to solve the problem at hand for at least a convex metallic body. Actually, we combine the general theory and the physical meanings nested in the mathematical model. The gradients of the objective function (the potential energy) in our model has a physical meaning; multiplying them by the negative sign we get a matrix $\mathbf{F}$ of electrostatic forces that particles exert on each other, see (Griffths, 1999) or (Ohanian, 2006). To make calculations easier in computing these forces, we denote $\vec{r}_{j}=\left[x_{j}, y_{j}, z_{j}\right]$. Then, the formula (1) can be rewritten as

$$
U\left(\vec{r}_{1}, \ldots, \vec{r}_{N}\right)=\frac{1}{2} \sum_{k=1}^{N} \sum_{j=1, j \neq k}^{N} \frac{q_{j} q_{k}}{\left[\left(x_{j}-x_{k}\right)^{2}+\left(y_{j}-y_{k}\right)^{2}+\left(z_{j}-z_{k}\right)^{2}\right]^{\frac{1}{2}}} .
$$

The force felt by a particle $p_{k}$ is given by

$$
\mathbf{F}_{k}=\left(-\frac{\partial U}{\partial x_{k}},-\frac{\partial U}{\partial y_{k}},-\frac{\partial U}{\partial z_{k}}\right)
$$

Where 


$$
\begin{aligned}
& \frac{\partial U}{\partial x_{k}}=-\sum_{j=1, j \neq k}^{N} \frac{q_{j} q_{k}\left(x_{k}-x_{j}\right)}{\left[\left(x_{j}-x_{k}\right)^{2}+\left(y_{j}-y_{k}\right)^{2}+\left(z_{j}-z_{k}\right)^{2}\right]^{\frac{1}{2}}}, \\
& \frac{\partial U}{\partial y_{k}}=-\sum_{j=1, j \neq k}^{N} \frac{q_{j} q_{k}\left(y_{k}-y_{j}\right)}{\left[\left(x_{j}-x_{k}\right)^{2}+\left(y_{j}-y_{k}\right)^{2}+\left(z_{j}-z_{k}\right)^{2}\right]^{\frac{1}{2}}}, \\
& \frac{\partial U}{\partial z_{k}}=-\sum_{j=1, j \neq k}^{N} \frac{q_{j} q_{k}\left(z_{k}-z_{j}\right)}{\left[\left(x_{j}-x_{k}\right)^{2}+\left(y_{j}-y_{k}\right)^{2}+\left(z_{j}-z_{k}\right)^{2}\right]^{\frac{1}{2}}},
\end{aligned}
$$

and then we get a matrix of forces $\mathbf{F}=\left[\mathbf{F}_{k}\right]_{1 \leq k \leq N}$. Since $\mathbf{F}_{k}$ is a $1 \times 3$ matrix, $\mathbf{F}$ is an $N \times 3$ matrix.

The strategy to solve the problem at hand relies on the fact that the displacement of the particles must be made within the surface $S$. So, the tangential component of the force felt by each particle is of great interest. The following is the mathematical framework to get these tangential components. Let $S$, the surface of the body, be given in Cartesian coordinates in the form $\Phi(x, y, z)=$ constant. Recall that the direction of $\nabla \Phi$, the gradient of $\Phi$, is always perpendicular to the surface $\Phi(x, y, z)=$ constant (Manfredo, 1976). Then, the tangential components $\mathbf{F}_{k T}$ are given by $\mathbf{F}_{k T}=$ $\mathbf{F}_{k}-\left(\mathbf{F}_{k} \cdot \mathbf{n}_{k}\right) \mathbf{n}_{k}$, where $\mathbf{n}_{k}$ is the outward unit vector normal to the surface $\mathrm{S}$ at the position $\vec{r}_{k}$ and derived from $\mathbf{n}=|\nabla \Phi|^{-1} \nabla \Phi$.

Suppose that the particle $p_{k}$ is at the position $\vec{r}_{k}^{(0)}$. Other particles exert a repulsive force $\mathbf{F}_{k}^{(0)}$ on it. Since the displacement must be made within the surface, we are interested in the tangential component of this force, $\mathbf{F}_{k T}^{(0)}$. Then the conceptual next position of the particle would be $\vec{r}_{k}{ }^{\prime}=\vec{r}_{k}^{(0)}+\alpha$ $\mathbf{F}^{(0)}{ }_{k T}$, where $\alpha$ is some positive number (with suitable units). But unfortunately this point is outside of the surface. We must therefore project the position $\vec{r}_{k}{ }^{\prime}$ to the surface and get the position $\vec{r}_{k}^{(1)}$. At the position $\vec{r}_{k}^{(1)}$ will happen the same scenario as that happened at $\vec{r}_{k}^{(0)}$ and we get the position $\vec{r}_{k}^{(2)}$, and so on. Now we need a recursive formula to enable us obtaining the position $\vec{r}_{k}^{(n+1)}$ from the position $\vec{r}_{k}^{(n)}$.

Suppose that the origin of coordinates is inside the body, if it is not the case a translation can do the job. Now choose a projection parallel to the vector $\vec{r}_{k}{ }^{\prime}$. In this case, the position $\vec{r}_{k}^{(1)}$ is such that the line joining the origin of coordinates to the position $\vec{r}_{k}{ }^{\prime}$ passes through the position $\vec{r}_{k}^{(1)}$. Therefore, it may be possible to scale the vector $\vec{r}_{k}{ }^{\prime}$ and get the desired 
position $\vec{r}_{k}^{(1)}$. Then we repeat the process until the convergence criterion is achieved.

\section{Illustration on ellipsoidal metallic bodies}

For example suppose that the surface $S$ of the body is an ellipsoid of equation $\frac{x^{2}}{a^{2}}+\frac{y^{2}}{b^{2}}+\frac{z^{2}}{c^{2}}=1$. Then $\Phi(x, y, z)=\frac{x^{2}}{a^{2}}+\frac{y^{2}}{b^{2}}+\frac{z^{2}}{c^{2}}$ and the

vector $\nabla \Phi=\left(\frac{2 x}{a^{2}}, \frac{2 y}{b^{2}}, \frac{2 z}{c^{2}}\right)$ is normal to the ellipsoid and from which we get $\mathbf{n}_{k}$, the unit vector normal to the

ellipsoid, at the position $\vec{r}_{k}$. This normal vector is used to determine $\mathbf{F}_{k T}$.

Moreover, the scaling of the vector $\vec{r}_{k}{ }^{\prime}$ to get the position $\vec{r}_{k}^{(1)}$ is made

possible by computing a scaling factor $t_{0, k}$ using the formula

$t_{0, k}=\left(\frac{\vec{r}_{k}^{\prime}(1)^{2}}{a^{2}}+\frac{\vec{r}_{k}^{\prime}(2)^{2}}{b^{2}}+\frac{\vec{r}_{k}^{\prime}(3)^{2}}{c^{2}}\right)^{\frac{1}{2}}$,

Where $\vec{r}_{k}{ }^{\prime}(1)=x_{k}^{\prime}, \vec{r}_{k}{ }^{\prime}(2)=y_{k}^{\prime}$ and $\vec{r}_{k}{ }^{\prime}(3)=z_{k}^{\prime}$. Hence the position is $\vec{r}_{k}^{(1)}$ obtained by

$\vec{r}_{k}^{(1)}=t_{0, k} \vec{r}_{k}{ }^{\prime}$ or equivalently

$\vec{r}_{k}^{(1)}=t_{0, k}\left(\vec{r}_{k}^{(0)}+\alpha \mathbf{F}_{k T}^{(0)}\right)$.

The formula (11) is generalized to give the recursive formula

$\vec{r}_{k}^{(n+1)}=t_{n, k}\left(\vec{r}_{k}^{(n)}+\alpha \mathbf{F}_{k T}^{(n)}\right)$,

in which

$t_{n, k}=\left(\frac{\vec{t}_{n, k}(1)^{2}}{a^{2}}+\frac{\vec{t}_{n, k}(2)^{2}}{b^{2}}+\frac{\vec{t}_{n, k}(3)^{2}}{c^{2}}\right)^{\frac{1}{2}}$,

with $\vec{t}_{n, k}=\left(\vec{r}_{k}^{(n)}+\alpha \mathbf{F}_{k T}^{(n)}\right)$. It is the recursive formula (12) that enable us to obtain the position $\vec{r}_{k}^{(n+1)}$ from the position $\vec{r}_{k}^{(n)}$.

\section{Numerical simulations}

Numerical simulations was done with a Matlab code implementing the formula (12). The stopping criterion we used is a non significant decrease in computed potential energy of order of $\varepsilon=10^{-8}$. In numerical experiments we considered bodies whose surfaces are ellipsoids and that have the same volume as the unit sphere. For those bodies the static equilibrium configuration has been determined. The corresponding potential energies 
have been also computed. We considered, first, the particular case of a unit sphere with an increasing number of charges $N$, where each charge gets smaller and smaller, in such a way that the total charge is fixed and equal to $Q=1$ esu. The discretization parameter being $q=Q / N$. Secondly, similar experiments were done considering the case of ellipsoids that have the same volume as the unit sphere. For example Figure 1 (left) shows the initial configurations of 1000 particles. The potential energy corresponding to this configurations is $U=0.50752833155242 \mathrm{erg}$. The static equilibrium configuration we get after running the code is shown in Figure 1 (right) and the corresponding potential energy is $U=0.48554272830599 \mathrm{erg}$. For an ellipsoid of parameters $b=c=0.8$ and $a=1 / b^{2}$, Figure 2 (left) shows the initial configurations of 640 particles whereas Figure 2 (right) shows the corresponding static equilibrium configuration. The potential energies are respectively $U=0.536633803810296 \mathrm{erg}$ and $U=0.459029324535688 \mathrm{erg}$. For an ellipsoid of parameters $b=c=0.5$ and $a=1 / b^{2}$, Figure 3 (left) shows the initial configurations of 1280 particles. The potential energy corresponding to this configuration is $0.743301809793660 \mathrm{erg}$. The obtained SEC is shown in Figure 3 (right) and the corresponding potential energy is $0.335945382140601 \mathrm{erg}$. Figure 3 (right) also shows an interesting physical feature; the particles are more concentrated on both tops of the ellipsoid than on the middle part as was expected.
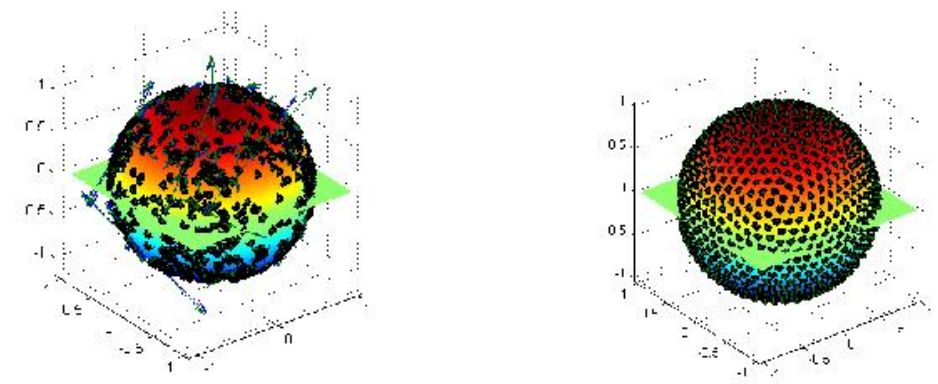

Figure 1: Initial configuration (left) and static equilibrium configuration (right) of 1000 particles on a unit sphere.
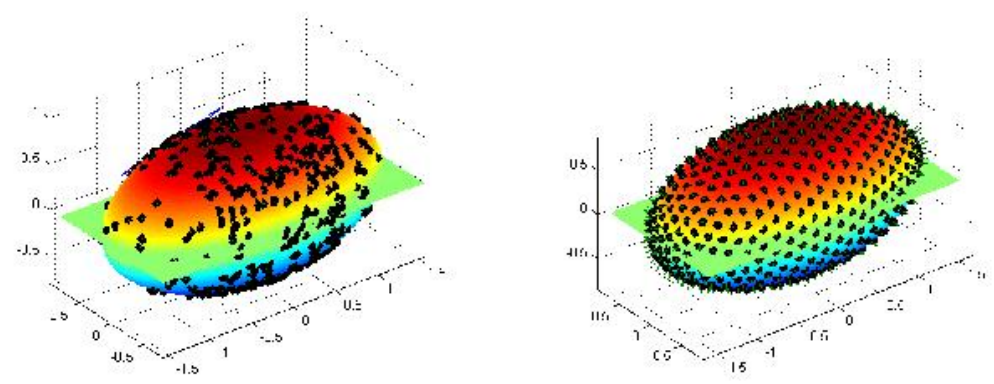
Figure 2: Initial configuration (left) and static equilibrium configuration (right) of 640 particles on an ellipsoid with parameters $b=c=0.8$ and $a=1 / b^{2}$.
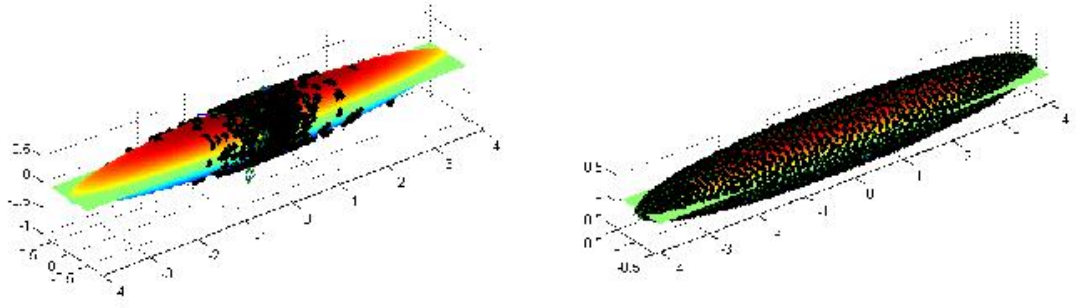

Figure 3: Initial configuration (left) and static equilibrium configuration (right) of 1280 particles on an ellipsoid with parameters $b=c=0.5$ and $a=1 / b^{2}$.

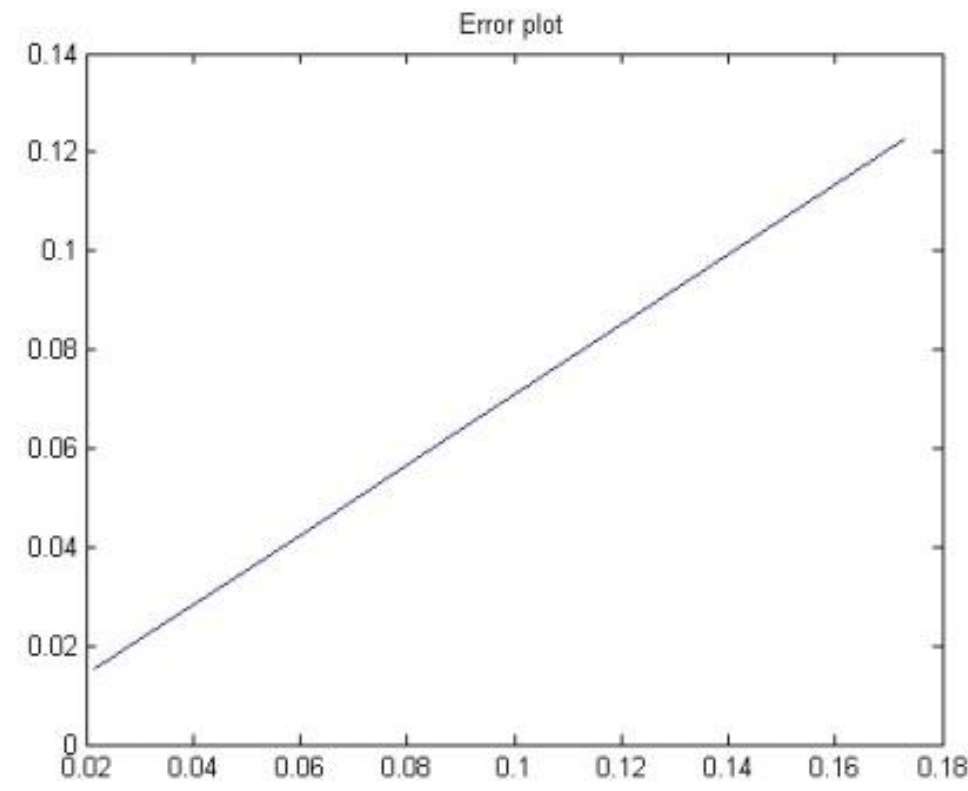

Figure 4: Plot of errors. 


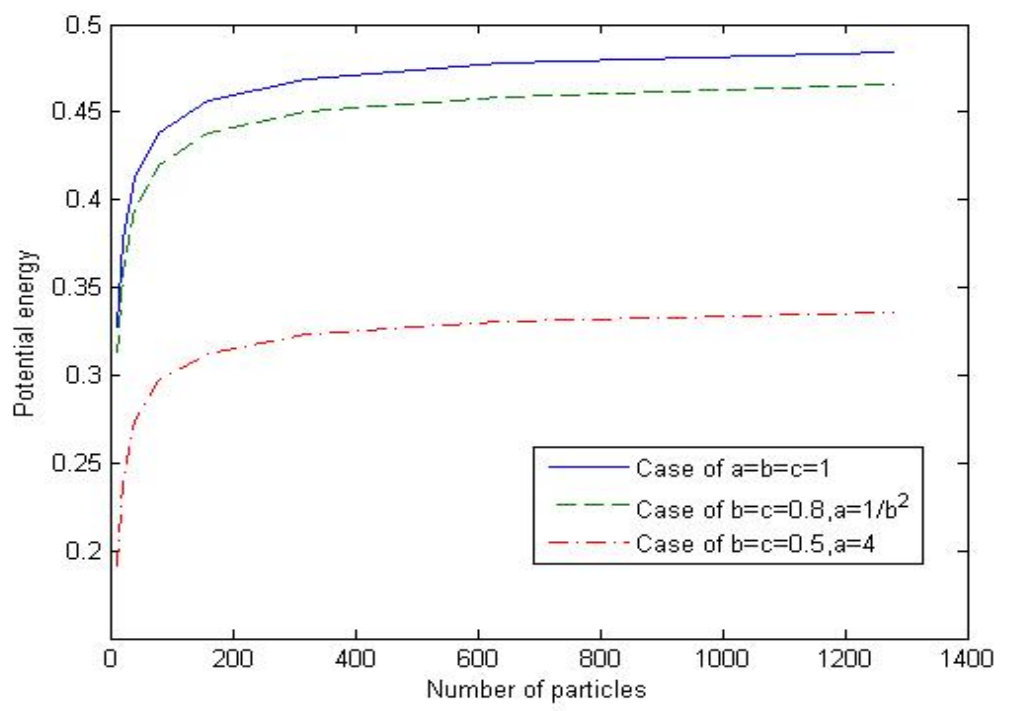

Figure 5: Comparison of potential energies according to eccentricity.

For the case of a sphere, the numerical results for the potential energy have been compared to the theoretical potential energy obtained using analytical methods. The potential energy (versus infinity) for a charged sphere with total charge $Q=1$ esu and radius $R=1 \mathrm{~cm}$, is given by the formula $U=Q^{2} /(2 \mathrm{R})$ (Baldassare, 1991), so that $U_{\text {ref }}=0.5 \mathrm{erg}$. It was observed that as the number of charges increases the potential energy at the static equilibrium approaches the theoretical potential energy. This is illustrated by Table 1 and proves that the techniques developed work as well. The errors are obtained by comparing the computed potential energies and the reference potential energy of $0.5 \mathrm{erg}$. Figure 4 is a plot of those errors, it shows that errors vary linearly. Therefore those techniques can be used where the analytical methods are hardly applicable or even where they fail. The case of ellipsoids is an example. For the case of ellipsoids we compared the computed values of the potential energies of ellipsoids that have the same volume as the unit sphere, increasing the eccentricity. We have remarked that the potential energy decreases as the eccentricity increases. This is illustrated by Table 2 and by Figure 5 . 
Table 1: Comparison of the computed potential energies of a unit sphere and the reference potential energy.

\begin{tabular}{lll}
\hline $\begin{array}{l}\text { Number of Particles } \\
N\end{array}$ & $\begin{array}{l}\text { Computed potential energy } \\
U_{\text {comp }}\end{array}$ & $\begin{array}{l}\text { Error } \\
\left|U_{\text {ref }}-U_{\text {comp }}\right|\end{array}$ \\
\hline 10 & 0.32717989915199 & 0.17282010084801 \\
20 & 0.37722534459517 & 0.12277465540483 \\
40 & 0.41297999662619 & 0.08702000337381 \\
80 & 0.43842174377579 & 0.06157825622421 \\
160 & 0.45643832882977 & 0.04356167117023 \\
320 & 0.46923577181013 & 0.03076422818987 \\
640 & 0.47825122480485 & 0.02174877519515 \\
1280 & 0.48463003137926 & 0.01536996862074 \\
\hline
\end{tabular}

Table 2: Comparison of potential energies according to eccentricity

\begin{tabular}{llll}
\hline $\begin{array}{l}\text { Number of } \\
\text { Particles }\end{array}$ & $\begin{array}{c}\text { Potential } \\
\text { Energy } \\
b=c=1\end{array}$ & $\begin{array}{c}\text { Potential } \\
\text { Energy }\end{array}$ & $\begin{array}{c}\text { Potential } \\
\text { Energy } \\
N=c=0.8\end{array}$ \\
& $a=1 / b^{2}$ & $a=1 / b^{2}$ & $b=c=0.5$ \\
& $U(\mathrm{erg})$ & $U(\mathrm{erg})$ & $a=1 / b^{2}$ \\
& 0.32717989915199 & 0.308764296082707 & 0.191201181093551 \\
10 & 0.37722534459517 & 0.359218046973656 & 0.240564688842096 \\
20 & 0.41297999662619 & 0.394877519494373 & 0.273914551023869 \\
40 & 0.43842174377579 & 0.419974849814247 & 0.296539450269219 \\
160 & 0.45643832882977 & 0.437683351948815 & 0.312131235348220 \\
320 & 0.46923577181013 & 0.450196835564463 & 0.322961835021825 \\
640 & 0.47825122480485 & 0.459029324535688 & 0.330600873801046 \\
1280 & 0.48463003137926 & 0.465287827998423 & 0.335945382140601 \\
\hline
\end{tabular}

\section{Concluding remarks}

This paper studied one of the problems related to electromagnetic phenomena, the static equilibrium configurations of charged metallic bodies. Whereas, in general, analytical methods fail to solve problems that are related to those electromagnetic phenomena especially for those that are directly applicable to real-world applications, we proposed a simple numerical method, easy to program, that can determine the static equilibrium configuration of a charged metallic body that is at least convex. The proposed method has some advantages; it combines the general theory and the physical meanings nested in the mathematical model. Most of methods for constrained nonlinear optimization problems accept only single column vectors as input variables. To apply them to the problem at hand, we need to reshape the matrices of positions and forces to suitable column vectors, a step which is not needed for the proposed method. This has a positive implication on the computational aspect as the number of charges gets more and more large. From simulation on a unit sphere, our results are in a well agreement with exact results obtained using analytical method. 


\section{References}

[1] Baldassare, D.B., 1991. Classical Theory of Electromagnetism, Prentice Hall, Englewood Cliffs, New Jersey 07632.

[2] Bondeson, A. et al., 2005. Computational Electromagnetics, Springer Science+Business Media, Inc, New York.

[3] Griffths, D.J., 1999. Introduction to Electrodynamics, Prentice-Hall, Inc., Upper Saddle River, New Jersey.

[4] Iusem A. N., 2003. On the convergence properties of the projected gradient method for convex optimization. Computational and Applied Mathematics, Vol. 22, N. 1, pp. 37-52.

[5] Manfredo, P. C., 1976. Differential Geometry of Curves and Surfaces, Prentice Hall, Inc., Englewood Cliffs, New Jersey.

[6] Nash, S.G. and Sofer, A., 2009. Linear and Nonlinear Programming, The McGraw-Hill Companies, Inc., Philadelphia.

[7] Ohanian, H.C., 2006. Classical Electrodynamics, Second Edition, INFINITY SCIENCE PRESS LLC, Hingham, Massachusetts.

[8] Wayne, M. S., 2002. Electricity, Magnetism and Light, Thomson learning inc., Toronto.

[9] Wenyu, S. and Ya-Xiang, Y., 2006. Optimization Theory and Methods: Nonlinear Programming, A Wiley-Interscience publication, Wiley \& Springer Science+Business Media, New York. 